\title{
Faster Use and Fewer Failures with Needle-Free Nasal Glucagon Versus Injectable Glucagon in Severe Hypoglycemia Rescue: A Simulation Study
}

\author{
Jean-François Yale, MD, Hélène Dulude, BPharm, $\mathrm{PhD}^{2}$ Marc Egeth, $\mathrm{PhD}^{3}$ Claude A. Piché, DVM, \\ Martin Lafontaine, BSc, Dolorès Carballo, BA, Rebecca Margolies, BSE, Emily Dissinger, MS, \\ Adam R. Shames, MBA, Nicole Kaplowitz, PhD, Michelle Xiaotian Zhang, MD, MBA, \\ Shuyu Zhang, MS, and Cristina B. Guzman, MD, MBA ${ }^{6}$
}

\begin{abstract}
Background: During severe hypoglycemic episodes, people with diabetes depend on others to help with treatment. We compared needle-free nasal glucagon and commercially available injectable glucagon for ease of use by caregivers of people with diabetes and by others in treating simulated episodes of severe hypoglycemia. Methods: Sixteen instructed caregivers and 15 noninstructed acquaintances administered nasal and injectable glucagon to manikins, simulating unconscious people with diabetes during severe hypoglycemia episodes.

Results: With nasal glucagon, 15 caregivers (94\%) and 14 acquaintances (93\%) administered a full dose (mean time 0.27 and $0.44 \mathrm{~min}$, respectively). One caregiver and one acquaintance did not administer nasal glucagon because they did not fully depress the plunger on the device. Two caregivers deliberately administered both insulin and nasal glucagon, believing that insulin would also help the patient. With injectable glucagon, eight caregivers (50\%) injected glucagon (mean time $1.89 \mathrm{~min}$ ), but only two (13\%) administered the full dose. Three acquaintances (20\%) injected a partial dose of injectable glucagon (mean time $2.40 \mathrm{~min}$ ); none gave a full dose. Errors included injecting diluent only, bending the needle, and injecting with an empty syringe. Two caregivers and one acquaintance injected insulin because they confused insulin with injectable glucagon.

Conclusions: More than $90 \%$ of participants delivered full doses of nasal glucagon, while $13 \%$ and $0 \%$ of caregivers and acquaintances delivered full doses of injectable glucagon, indicating that nasal glucagon is easier for nonmedically trained people to administer. Thus, nasal glucagon has the potential to substantially improve treatment for patients experiencing a life-threatening episode of severe hypoglycemia.
\end{abstract}

Keywords: Nasal glucagon, Injectable glucagon, Hypoglycemia, Human factors.

\footnotetext{
${ }^{1}$ McGill Department of Medicine, McGill University Health Center, McGill University, Montréal, Québec, Canada.

${ }^{2}$ Locemia Solutions, Montréal, Canada.

${ }^{3}$ Core Human Factors, Inc., Bala Cynwyd, Pennsylvania, PA.

${ }_{5}^{4}$ Formerly Core Human Factors, Inc., Bala Cynwyd, Pennsylvania, PA.

${ }^{5}$ Eli Lilly Canada, Inc., Toronto, Ontario, Canada.

${ }^{6}$ Eli Lilly and Company, Indianapolis, IN.

Parts of this study have been presented in abstract form at the 51st Annual Meeting of the European Association for the Study of Diabetes (EASD), Stockholm, Sweden, September 14-18, 2015; and at the 10th International Conference on Advanced Technologies and Treatments for Diabetes (ATTD), Paris, France, February 15-18, 2017.

(C) Jean-François Yale, et al., 2017; Published by Mary Ann Liebert, Inc. This Open Access article is distributed under the terms of the Creative Commons Attribution Noncommercial License (http://creativecommons.org/licenses/by-nc/4.0/) which permits any noncommercial use, distribution, and reproduction in any medium, provided the original author(s) and the source are credited.
} 


\section{Introduction}

$\mathbf{U}$ NTREATED, SEVERE HYPOGLYCEMIA CAN lead to cognitive dysfunction, convulsions, coma, and death. ${ }^{1}$ Fear of severe hypoglycemia can lead patients toward glycemic control favoring hyperglycemia and related complications. ${ }^{2,3}$ Over $60 \%$ of family members of people with diabetes are worried about the risk of hypoglycemic events. ${ }^{4}$

The prevalence of severe hypoglycemic events suggests this concern is warranted. United States 2011 data showed 282,254 visits to hospital emergency rooms with a first-listed diagnosis of hypoglycemia. 5 T1D Exchange data in the United States indicated that $6 \%$ of participants surveyed had experienced hypoglycemia-related seizure or loss of consciousness during a 3-month period. ${ }^{6}$ Among participants with $\geq 50$ years duration of type 1 diabetes (T1D), the rate was $8 \%{ }^{6}$ Similar results were found in a 24 -country study, $14.4 \%$ of 8022 people with T1D and $8.9 \%$ of 19,563 insulin-using people with T2D reported $\geq 1$ severe hypoglycemic episode during a 4-week period. ${ }^{7}$

Injectable glucagon is the only treatment available outside of a clinical setting for people who experience severe hypoglycemia and cannot ingest carbohydrates. Currently available glucagon kits contain a vial of powdered glucagon, a syringe prefilled with diluent, and instructions for reconstitution/administration of the glucagon. ${ }^{1}$ Some caregivers of people with diabetes may be familiar with the use of needles and syringes, but others may not. Moreover, fear of needles is common ${ }^{8-10}$ and could prevent effective administration of injectable glucagon by some people. For people without medical training, the multistep reconstitution and injection procedure might be complex and daunting, with substantial risk of errors. ${ }^{11}$ Thus, another mode of glucagon delivery may be desirable.

A survey concerning coping strategies for hypoglycemia found that most patients with insulin-dependent diabetes would prefer intranasally-administered glucagon if it were available $(67 \%)$ and believed the people surrounding them would prefer to administer glucagon intranasally $(82 \%) .^{12}$ The study concluded that intranasal administration would increase glucagon use and decrease the need for emergency medical services. ${ }^{12}$

Nasal Glucagon is a dry powder synthetic glucagon that is being developed for the treatment of severe hypoglycemia as a single-dose drug combination device, allowing simple, one- step, needle-free administration. ${ }^{13}$ A meta-analysis of five studies comparing nasally-administered glucagon and injectable glucagon showed they were similarly effective for treatment of hypoglycemia. ${ }^{14}$ Nasal glucagon showed noninferiority to injectable glucagon for treatment of severe hypoglycemia in adults with T1D. ${ }^{15}$ It was also studied in children with T1D in a controlled clinical setting ${ }^{16}$ and in adults and children in the real world. ${ }^{17,18}$

Ease of use of nasal glucagon and injectable glucagon has not been previously assessed. This study compared the ability of people without medical training to administer nasal glucagon and injectable glucagon during simulated severe hypoglycemia episodes. Ease of use, speed, effectiveness of dose delivery, and participant preference were assessed during simulated emergency situations in which a manikin represented a person who was unconscious due to severe hypoglycemia.

\section{Research Design and Methods}

This study, AMG111, was approved by a local institutional review board. Treatment options (Fig. 1) were a needle-free nasal glucagon rescue device (Nasal Glucagon, Eli Lilly and Company) and a commercially-available injectable glucagon emergency kit (Eli Lilly and Company). Both glucagon treatments had illustrations on the packaging showing how to deliver the glucagon (Supplementary Appendices 1 and 2; Supplementary Data are available online at www.liebertpub.com/dia).

\section{Study participants}

People in a large U.S. metropolitan area who had previously expressed interest in taking part in research studies were screened by telephone interview for factors including age, experience with diabetes, and knowledge of glucagon. Before study entry, participants had no training or experience in administering glucagon. All participants signed informed consent forms. Two different groups of participants were recruited for this study. Group 1 comprised pairs (people with diabetes and their primary caregivers), who participated in three study sessions (Table 1). Group 2 comprised individuals who had no experience with glucagon and diabetes, but would try to help if an acquaintance of theirs experienced a severe hypoglycemic episode; they participated in only one study session (Table 2). Individuals in this second group are referred to as "acquaintances" throughout this article. They

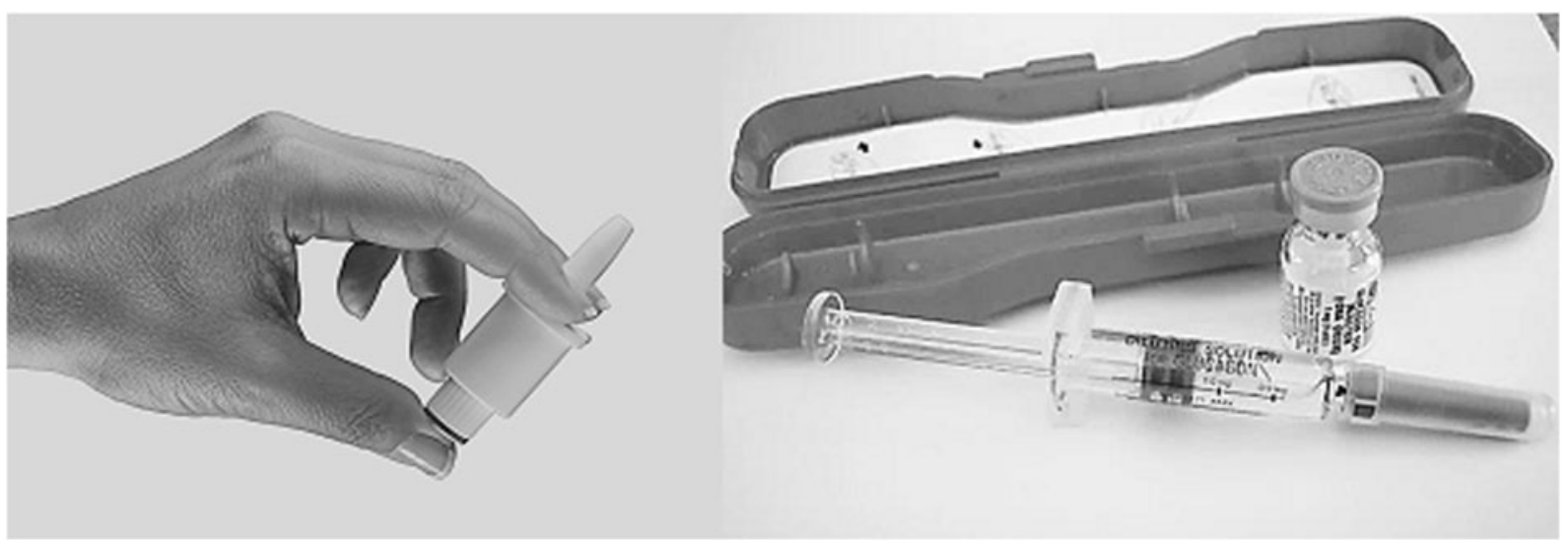

FIG. 1. Nasal glucagon spray device (left) and injectable glucagon kit (right). 
Table 1. Summary of the Three Sessions Conducted with Group 1

(People with Diabetes and Their Caregivers)

\begin{tabular}{|c|c|c|c|c|}
\hline Session 1 (60 min) & & Session 2 (60 min) & & Session 3 (30 min) \\
\hline $\begin{array}{l}\text { Person with diabetes and } \\
\text { caregiver are introduced } \\
\text { to the study and sign } \\
\text { consent forms }\end{array}$ & $\begin{array}{c}1-2 \text { week } \\
\text { delay }\end{array}$ & $\begin{array}{l}\text { Person with diabetes learns } \\
\quad \text { glucagon treatment option } 2^{\mathrm{a}}\end{array}$ & $\begin{array}{l}1-2 \text { week } \\
\text { delay }\end{array}$ & $\begin{array}{l}\text { Caregiver uses glucagon } \\
\text { treatment option } 2 \text { with } \\
\text { a manikin in a simulated } \\
\text { emergency situation }\end{array}$ \\
\hline $\begin{array}{l}\text { Person with diabetes learns } \\
\text { glucagon treatment } \\
\text { option } 1^{\mathrm{a}}\end{array}$ & & $\begin{array}{l}10 \text { - to } 30 \text {-min delay for person } \\
\text { with diabetes }\end{array}$ & & $\begin{array}{l}\text { Person with diabetes } \\
\text { and caregiver are } \\
\text { interviewed regarding }\end{array}$ \\
\hline $\begin{array}{l}\text { 30-min delay for person } \\
\text { with diabetes }\end{array}$ & & $\begin{array}{l}\text { Caregiver uses glucagon } \\
\text { treatment option } 1 \text { with a } \\
\text { manikin in a simulated } \\
\text { emergency situation, }\end{array}$ & & overall preferences \\
\hline $\begin{array}{l}\text { Person with diabetes } \\
\text { instructs the caregiver } \\
\text { on how to use glucagon } \\
\text { treatment option }\end{array}$ & & $\begin{array}{l}\text { 10- to } 30 \text {-min delay for caregiver } \\
\text { to recuperate from glucagon } \\
\text { treatment option } 1 \\
\text { administration } \\
\text { Person with diabetes instructs the } \\
\text { caregiver on how to use } \\
\text { glucagon treatment option } 2\end{array}$ & & \\
\hline
\end{tabular}

${ }^{a}$ Option 1 is the first glucagon treatment used by the pair (either nasal glucagon or injectable glucagon depending on randomization). Option 2 is the second treatment used by the pair.

did not necessarily know anyone with diabetes; however, before each simulation, they were provided with information that an acquaintance of a person with diabetes might have: the simulated patient has diabetes and an appropriate treatment could be found in the patient's backpack.

\section{Order of treatment option use}

Pairs and participants were numbered sequentially based on when they first came to the study site. All odd-numbered pairs and odd-numbered acquaintances used injectable glucagon first; all even-numbered pairs and acquaintances used nasal glucagon first.

Table 2. Summary of the Single Session Conducted With Group 2 (ACQuaintances)

Acquaintance session (40 min)

Acquaintance is introduced to the study and signs consent form

Acquaintance learns of glucagon treatment option $1^{\mathrm{a}}$, but receives no instruction about it

Acquaintance completes a short distractor task (a "memory decay" period)

Acquaintance uses glucagon treatment option 1 with a manikin in a simulated emergency situation

10-min break

Acquaintance learns of glucagon treatment option $2^{\mathrm{a}}$, but receives no instruction about it

Acquaintance completes a short distractor task (a "memory decay"')

Acquaintance uses glucagon treatment option with a manikin in a simulated emergency situation

Acquaintance is interviewed regarding overall preferences

\footnotetext{
${ }^{a}$ Option 1 is the first glucagon treatment used (either nasal glucagon or injectable glucagon depending on randomization). Option 2 is the second treatment used.
}

Group 1, pairs: people with diabetes and their caregivers

At the first session, (Table 1) to simulate what might happen in a healthcare provider's office, people with diabetes were given step-by-step instructions on how to use one of the glucagon treatment options. The instructions for use (IFU) for injectable glucagon were those provided with the commercially available product (Supplementary Appendix 1), while the IFU for the nasal glucagon were instructions drafted for use in this study (Supplementary Appendix 2). Caregivers were not present during the training. After a 10- to 30-min memory decay period, the people with diabetes were asked to have a discussion with their caregivers, such as they might have in real life after receiving the glucagon treatment option. There was then a 1- to 2-week memory decay period before the second session when caregivers were assessed on their ability to use the first glucagon treatment in the simulated emergency situation. At the second session, people with diabetes were instructed about the other glucagon treatment option without the presence of their caregivers, and after the 10- to 30-min for memory decay, they discussed that treatment option with their caregivers. At the third session, caregivers were assessed on their ability to use the second glucagon treatment in the simulated emergency situation (Table 1).

Instruction, group 1 (pairs: people with diabetes and their caregivers)

At sessions 1 and 2, we assessed how well the people with diabetes instructed their caregiver about administering the glucagon treatment by recording which steps of the procedure were described to the caregiver and the total time needed to teach the steps (starting when the person with diabetes began teaching and ending when the pair indicated to the moderator that they were finished). 


\section{Group 2 (acquaintances)}

Each acquaintance participated in a single session, which included two simulated emergency situations, one with each glucagon treatment option (Table 2). Acquaintances received no instruction in administering either type of glucagon; therefore, there was no assessment of instruction for this group. Instead, acquaintances were only shown what the relevant glucagon treatment looked like before beginning each simulation. Acquaintances then completed a brief "distractor" task to allow memory decay, before participating in a severe hypoglycemia simulation with that glucagon treatment. During the simulations, acquaintances had access to the relevant IFUs (Supplementary Appendices 1 and 2).

\section{Simulated severe hypoglycemia episodes (group 1 caregivers and group 2 acquaintances)}

In these simulations, a fully clothed, adult-sized manikin represented a person with diabetes who was unconscious due to a severe hypoglycemic episode. The manikin and a backpack were placed on the floor. The backpack contained a jacket, the relevant glucagon treatment, and a pouch containing diabetes supplies that an insulin user might carry (glucometer and strips, alcohol swabs, lancing device, insulin syringe, and a vial of insulin). Caregivers and acquaintances were instructed to administer the glucagon treatment as quickly as possible and told that the necessary supplies were in the backpack. Distracting sounds and other stressors were used to model the urgency and stress of a real-life emergency. Sounds included beeping noises of increasing volume and intensity, a ringing telephone, and a person knocking on the door and interrupting the session to say that an ambulance for the "patient" would not arrive for $15 \mathrm{~min}$. Other stressors included the moderator reminding the participants that the sessions were being watched and videotaped, they were being timed with a stopwatch, their performance will be evaluated, and the situation was urgent. The moderator remained in the room throughout the simulation.

\section{Assessments of glucagon administration (group 1 caregivers and group 2 acquaintances)}

Glucagon administration was assessed by recording which steps from the IFUs were correctly performed by the caregivers or acquaintances, as well as the time taken to administer each glucagon treatment (timing began when the participants found the glucagon treatment in the backpack and ended when glucagon administration was complete). Caregivers and acquaintances were interviewed after each simulation to determine the reasons for failures to complete any of the steps involved in administering the glucagon treatment.

\section{Assessment of glucagon dose delivered (group 1 caregivers and group 2 acquaintances)}

For injectable glucagon, the percentage of dose administered was assessed by comparing weight of each participant's syringe, needle, and vial before and after administration. Thus, the calculated percentage represents the maximum percentage that may have been given, since some medication could have been lost before the injection (e.g., injected into the air). For nasal glucagon, full depression of the plunger (such that the colored line on the device is no longer visible) causes delivery of the entire dose, while only partially depressing the plunger (colored line still visible) does not release any glucagon. Therefore, when participants fully depressed the plunger, delivery of a full dose of glucagon was recorded. When participants did not fully depress the plunger, it was recorded that they delivered $0 \%$ of the glucagon dose.

\section{Questionnaires and assessment scales}

Study participants (pairs and acquaintances) completed the Rapid Estimate of Adult Health Literacy (REALM) to assess participants' understanding of common healthcare terms. ${ }^{19}$ The Stanford Health Assessment Questionnaire Disability Index (HAQ-DI), which assesses eight categories of functioning: dressing, rising, eating, walking, hygiene, reach, grip, and usual activities, ${ }^{20}$ was used primarily as a memory decay/distractor task. People with diabetes and caregivers assessed ease of instruction concerning each glucagon treatment option, using the NASA Task Load Index (TLX), which includes visual analog scales for assessing mental demand, physical demand, temporary demand, performance, effort, and frustration. ${ }^{21}$ Also, after each simulation, caregivers and acquaintances assessed the complexity of administering the glucagon treatment using the NASA TLX. Participants were also asked questions specific to the glucagon treatment that they had just used (Supplementary Appendix 3). People with diabetes answered additional questions about giving instruction to the caregivers (Supplementary Appendix 3).

\section{Sample size}

To achieve a statistical power $\geq 95 \%$, assuming a true within-subject mean difference of $\geq 40$ s (with standard deviation of $40 \mathrm{~s}$ ) between glucagon treatments in time to dose delivery using an alpha of 0.05 (two sided), 16 pairs and 16 acquaintances would be required to complete the study. We estimated a $20 \%$ dropout rate, and therefore planned to recruit 20 pairs and 20 acquaintances.

\section{Statistical method}

Statistical software used was SAS version 9.4 (SAS Institute, Inc., Cary, NC). Fisher's exact test was used to assess the difference between nasal glucagon and injectable glucagon for binary outcome. Within-subject paired $t$-test was used to assess the difference between nasal glucagon and injectable glucagon for continuous outcome.

\section{Results}

Nineteen pairs, consisting of people with diabetes and their caregivers, were recruited for Group 1 (Fig. 2); 16 pairs completed the study. Two pairs dropped out for personal reasons. The caregiver in another pair indicated that, due to her own disabilities, she would call 911 rather than attempt to help her partner during an episode of severe hypoglycemia. As a result, she did not attempt the simulated glucagon treatment and the pair was excluded from the study.

Twenty individuals were recruited for Group 2 (the acquaintances' simulations); 15 acquaintances completed the study (Fig. 2). Five acquaintances failed to keep appointments or dropped out of the study. Fifteen acquaintances 

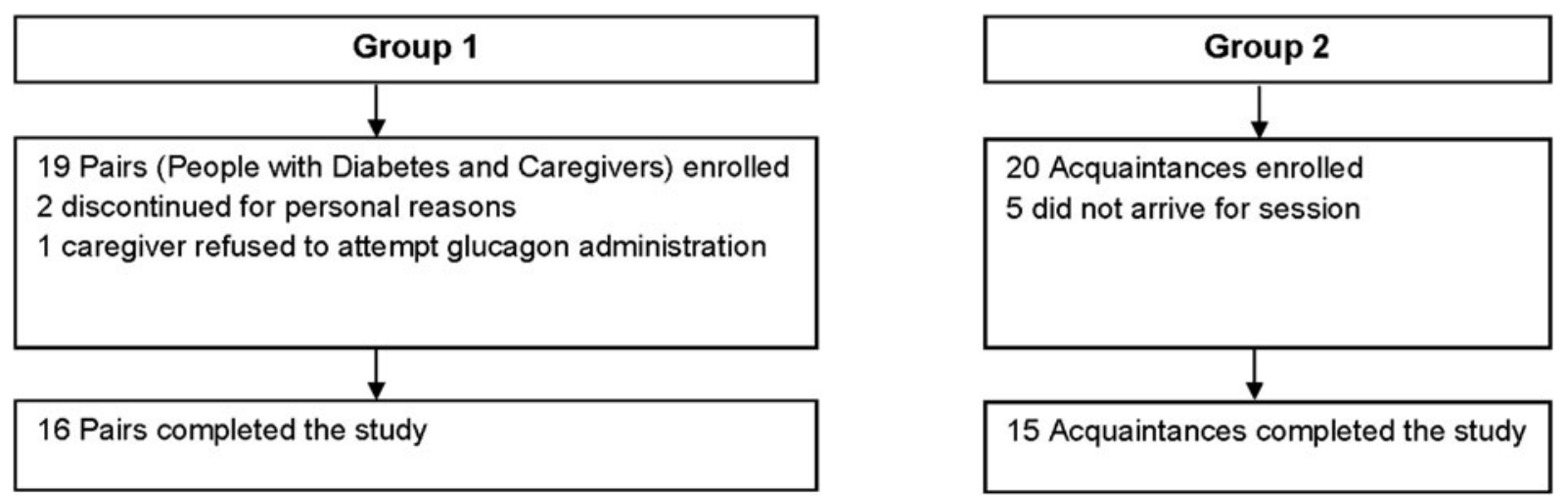

FIG. 2. Participant flow diagram.

completed the study. Because there were fewer acquaintances than originally planned, the statistical power for analyses of acquaintances was $94 \%$ rather than $95 \%$.

People with diabetes, caregivers, and acquaintances were all predominantly female (Table 3 ). Acquaintances were younger, on average, but had an age range comparable to the other participants. Two people with diabetes, four caregivers, and three acquaintances were aged 65 or older. Median duration of diabetes for people with diabetes was 15 years (Table 3).

\section{Group 1 simulation (pairs: people with diabetes and their caregivers) \\ Instruction}

Mean instruction time was 7.2 min for injectable glucagon versus $2.6 \mathrm{~min}$ for nasal glucagon. IFU of injectable glucagon contained 14 key points (Supplementary Table S1). Of these 14 steps, only 2 were communicated by all people with diabetes $(100 \%)$ when they were instructing their caregivers. These two steps were to inject the entire contents of the syringe into the glucagon vial and swirl the bottle gently until the glucagon dissolves completely. The least commonly taught point, to press an alcohol swab against the injection site following the injection, was taught by only $19 \%$ people with diabetes (Supplementary Table S1).

IFU of nasal glucagon contained eight key points (Supplementary Table S1). Three of these were included by all persons with diabetes $(100 \%)$ when teaching their caregivers about nasal glucagon: removing the spray device from the packaging tube, holding the spray device between thumb and fingers, and inserting the spray device into one of the patient's nostrils. The least commonly taught point, a warning not to test the device before using, was taught by $31 \%$ people with diabetes (Supplementary Table S1).

Glucagon administration by caregivers (from group 1) in a simulated emergency situation

\section{- Injectable glucagon}

None of the individual steps needed for administration of injectable glucagon were successfully completed by $100 \%$ of the caregivers (Supplementary Table S1). The most commonly completed step was removing the needle protector from the syringe $(88 \%)$. The least commonly completed steps were withdrawing all the solution from the bottle (13\%), cleaning the injection site (13\%), using an injection site on the buttocks, arm, or thigh (13\%), inserting the needle into loose tissue (13\%), and pressing an alcohol swab against the injection site following the injection (0\%). Root causes for not wiping the rubber stopper with an alcohol swab, cleaning the injection site, or pressing an alcohol swab against the injection site were that participants did not see these directions in the IFU, could not find the alcohol swabs, forgot, or were in a hurry. Causes for not injecting into the buttocks, arm, or thigh were that the caregiver was used to injecting into a different site or a belief that the wrist was a better site with more blood vessels (Supplementary Table S1).

Only two caregivers successfully reconstituted the glucagon and injected the full, recommended dose into the manikin. Another six caregivers injected partial doses of glucagon. The calculated maximum amounts delivered to the manikin were $3 \%, 43 \%, 51 \%, 58 \%, 81 \%$, and $85 \%$ of the full dose. However, the delivered doses may have been even smaller if the caregiver primed the syringe, releasing some glucagon into the air. On average, it took these caregivers $1.89 \mathrm{~min}$ (range 1.3-2.75 min) to deliver these full or partial doses (Table 4 and Fig. 3). Half the caregivers delivered no

Table 3. Participant Demographics

\begin{tabular}{lccc}
\hline & \multicolumn{2}{c}{ Group 1 } & $\begin{array}{c}\text { Group 2 } \\
\text { Acquaintances } \\
(\mathrm{N}=15)\end{array}$ \\
\cline { 2 - 3 } & $\begin{array}{c}\text { People with diabetes } \\
(\mathrm{N}=16)\end{array}$ & $\begin{array}{c}\text { Caregivers } \\
(\mathrm{N}=16)\end{array}$ & $10(63)$ \\
Female, $n(\%)$ & $12(75)$ & $9(60)$ \\
Median age, years (minimum, maximum) & $57(26,76)$ & $54(20,69)$ & $40(22,78)$ \\
Median diabetes duration, years (minimum, maximum) & $15(2,39)$ & Not applicable & Not applicable \\
\hline
\end{tabular}




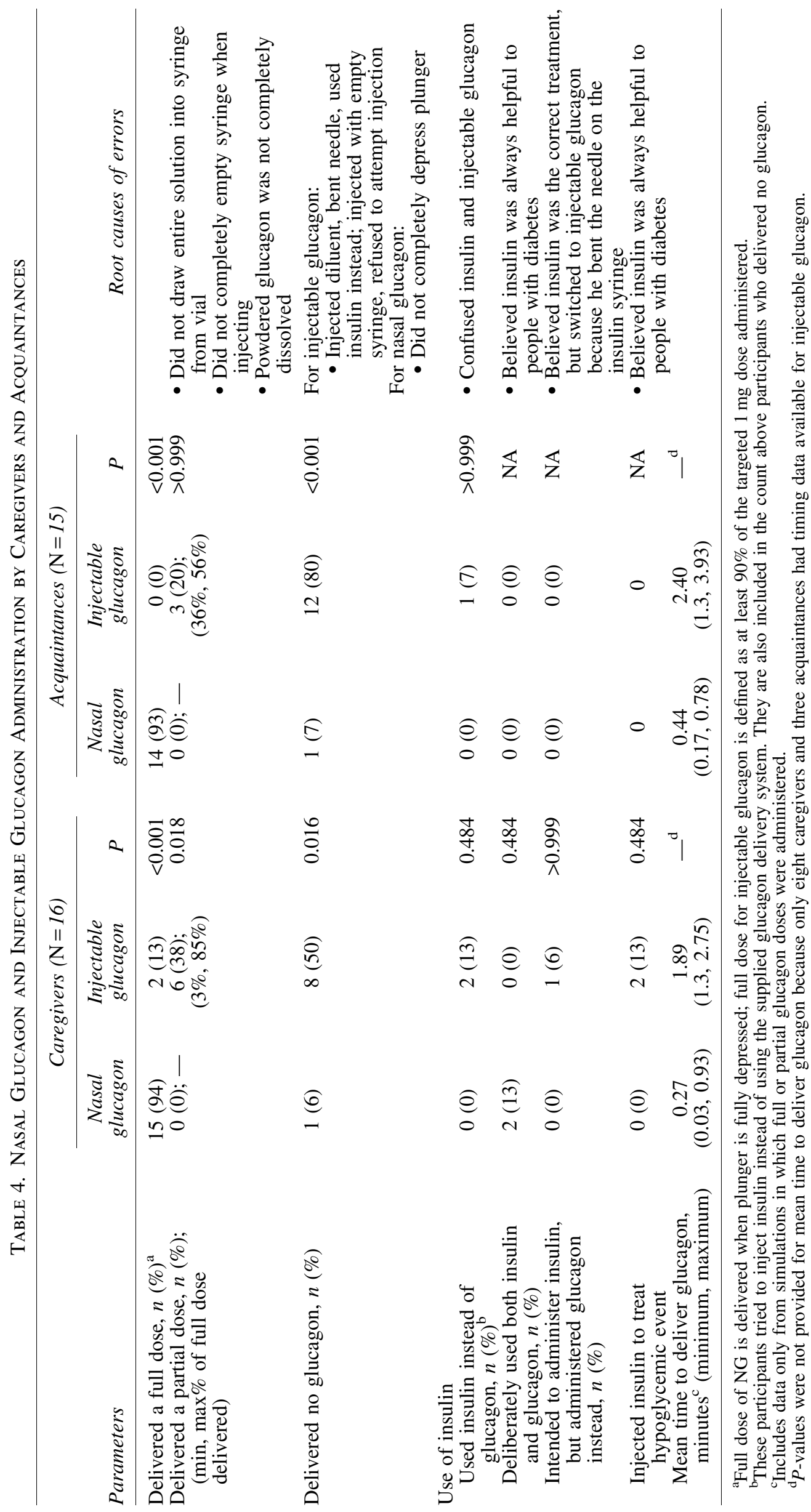




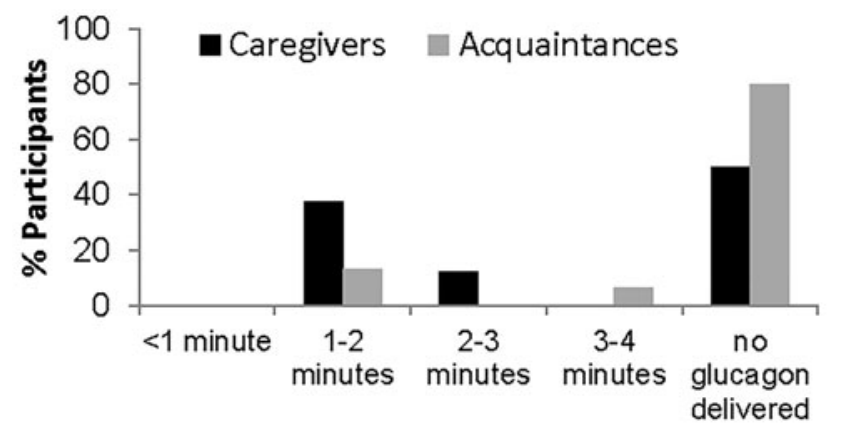

Time to Administer Injectable Glucagon

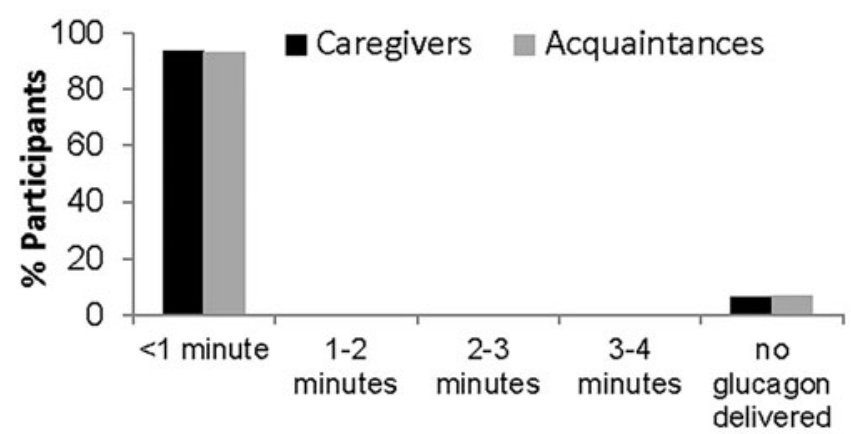

Time to Administer Nasal Glucagon

FIG. 3. Time to administer injectable glucagon and nasal glucagon.

injectable glucagon to the manikin because they injected only diluent, used insulin instead, or bent the needle (Table 4).

Overall, five caregivers attempted to inject the manikin with insulin during the simulation with injectable glucagon. Two caregivers injected insulin instead of glucagon because they confused injectable insulin and glucagon due to the similar form factor. These two caregivers were told by the moderator that they had injected insulin rather than glucagon, and were then asked to inject the glucagon. One injected diluent and one injected $42 \%$ of the dose into the manikin. Because these caregivers injected insulin first and then had to be prompted to inject glucagon, their times were not included in the calculation of the mean time to administer glucagon shown above. One caregiver believed insulin was the correct treatment, but switched to injectable glucagon because he bent the insulin needle. Finally, two other caregivers intentionally injected the manikin with insulin; one thought insulin was the correct treatment for a hypoglycemic event and one believed that all diabetes medication was insulin.

\section{- Nasal glucagon}

All caregivers (100\%) were able to perform seven of the eight steps necessary for administering nasal glucagon. One caregiver failed to depress the plunger fully (Supplementary Table S1), and thus no glucagon was delivered to the manikin (Supplementary Table S1 and Fig. 3). The 94\% of caregivers who were able to deliver a full dose of nasal glucagon, did so with a mean time of $0.27 \mathrm{~min}$ or about $16 \mathrm{~s}$ (Table 4). All doses were administered in less than a minute (range 0.03-0.93 min; Fig. 3 and Table 4). Two caregivers who successfully delivered nasal glucagon to the manikin then also deliberately administered insulin as well, believing that insulin was always an appropriate treatment for people with diabetes (Table 4).

\section{Group 2 (acquaintances simulation)}

\section{- Injectable glucagon}

Of the 14 steps listed for administering injectable glucagon correctly (Supplementary Table S1), none was completed by $100 \%$ of acquaintances. The most commonly completed steps were removing the needle protector from the syringe (93\%), inserting the needle into loose tissue and injecting all of the solution (67\%), and removing the flip-off seal from the bottle $(60 \%)$. All other steps were completed by $20 \%$ or fewer of acquaintances (Supplementary Table S1). Many acquaintances failed to reconstitute the glucagon because they assumed the diluent in the syringe was the needed medication, or they bent the syringe needle trying to inject the diluent into the vial without properly removing the cap from the vial. One acquaintance did reconstitute the glucagon, but was unable to figure out how to transfer it back to the syringe (Supplementary Table S1).

None of the acquaintances delivered a full dose of injectable glucagon (Table 4). Only three delivered partial doses: $55.9 \%$ of dose delivered in $3.9 \mathrm{~min}, 35.6 \%$ of dose delivered in $2.0 \mathrm{~min}$, and an indeterminate dose delivered in $1.3 \mathrm{~min}$ (Fig. 3). This last dose could not be determined because wet powder remained in the glucagon vial at the end of the simulation, indicating that glucagon was not fully reconstituted. Of the 12 acquaintances who did not administer any injectable glucagon, 9 (60\% of all acquaintances) injected diluent, believing it was the needed medication. One acquaintance refused to attempt the injection and said she would wait for an ambulance, one injected the manikin with an empty syringe, and one injected the manikin with insulin instead. This last acquaintance, when told by the moderator to use the injectable glucagon, injected the manikin with diluent.

\section{- Nasal glucagon}

All acquaintances (100\%) performed seven of the eight steps necessary for administering nasal glucagon. One acquaintance was unaware of the requirement to push the plunger until the colored band was no longer visible, did not depress the plunger fully, and thus did not deliver any glucagon to the manikin (Table 4). A full dose of nasal glucagon was delivered by $93 \%$ of acquaintances, within a mean time of 0.44 min or about $26 \mathrm{~s}$ (Table 4 and Fig. 3). No acquaintances administered insulin during the simulation with nasal glucagon.

\section{Participants' preferences and assessments concerning glucagon treatments}

Caregivers were asked whether they experienced fear or hesitation when administering glucagon during the simulated hypoglycemic event; 4 (25\%) answered yes about nasal glucagon and $8(50 \%)$ answered yes about injectable glucagon. Four (27\%) acquaintances reported experiencing fear or hesitation when using nasal glucagon compared to $9(60 \%)$ for injectable glucagon. The NASA TLX was used to assess the complexity of administering glucagon after each simulation; lower numbers indicate less complexity. Caregivers 
rated nasal glucagon as 43.2 compared to injectable glucagon as $68.9(P<0.05)$. Acquaintances rated nasal glucagon as 51.8 and injectable glucagon as $65.6(P<0.05)$.

People with diabetes were asked which glucagon treatment they would prefer that their friends or family use. Eleven $(69 \%)$ preferred nasal glucagon to injectable glucagon. They thought the nasal glucagon rescue device was easier to use, easier to teach, and easier to carry with no chance of needle breakage or accidental needle stick. Three (19\%) preferred injectable glucagon over nasal glucagon stating that needles were better than nasal sprays, and their families were already comfortable with needles. Two (13\%) did not state a preference.

When asked which treatment option they would recommend, $13(81 \%)$ caregivers preferred nasal glucagon to injectable glucagon; the predominant reasons were that nasal glucagon was easier and less stressful to use, and less embarrassing. One $(6 \%)$ caregiver had no preference. Two $(13 \%)$ caregivers preferred injectable glucagon; one believed injected glucagon would be more effective and one preferred injectable glucagon because, although nasal glucagon was easier to administer, the syringe made it easier to see that the dose had been administered. Interestingly, neither of the caregivers who preferred injectable glucagon had been able to administer a full dose of injectable glucagon during the simulation.

All acquaintances (100\%) stated that they would recommend nasal glucagon, but not injectable glucagon; reasons included the ease of use of nasal glucagon and the fact that needles are intimidating.

\section{Discussion}

Nasal glucagon shows promise in addressing the unmet need for a needle-free, easy-to-administer form of glucagon. IFU of nasal glucagon appear to be easier to teach than IFU of injectable glucagon. Moreover, the acquaintances (93\%) were as successful as the instructed caregivers (94\%) in administering nasal glucagon and were almost as quick as caregivers in time to administration (mean time 0.44 min vs. $0.27 \mathrm{~min}$, compared to $2.4 \mathrm{~min}$ and $1.89 \mathrm{~min}$ with injectable glucagon), showing that successful nasal delivery was at or near ceiling levels even without previous instruction.

One caregiver and one acquaintance did not understand that it was necessary to depress the plunger on the device until the colored line was no longer visible. Therefore, they delivered no glucagon to the manikin. However, in real-life use, the visibility of the colored line would indicate that the device was still usable. If, during an emergency, the first attempt to deliver nasal glucagon failed because the plunger was not depressed completely, a second, potentially-successful attempt could be made.

In contrast, injectable glucagon was much more difficult for study participants to administer. Only two instructed caregivers $(13 \%)$ were able to deliver $100 \%$ of the full recommended dose of injectable glucagon $(P<0.001$ vs. nasal glucagon). No acquaintances $(0 \%)$ delivered a full dose of injectable glucagon $(P<0.001$ vs. nasal glucagon). The process of reconstituting glucagon was unfamiliar to both caregivers and acquaintances, and caused many difficulties. In addition, some caregivers and acquaintances found needles intimidating to use, and one refused to attempt an in- jection. Failures to use injectable glucagon often left the device in an unusable state, for example, with the diluent used or the needle on the syringe bent.

Insulin was available to participants during these simulations because it is likely that an insulin-using person with diabetes would have their insulin supply nearby during an actual severe hypoglycemic episode. Some caregivers and acquaintances confused injectable insulin and injectable glucagon, and injected insulin by mistake. No one confused nasal glucagon with injectable insulin, due to the different forms of the two products. This suggests that the risk of accidentally delivering insulin during hypoglycemic episodes can be reduced by using a glucagon that differs from insulin in route of administration and dosage form.

The inclusion of insulin in the design of the study also brought to light the need for better education about hypoglycemia and the effects of insulin. Two caregivers intentionally administered both nasal glucagon and insulin during these simulations because they mistakenly believed that insulin would regulate blood sugar, or because they were used to giving insulin to treat diabetes and did not distinguish between hyperglycemia and hypoglycemia.

In this study, four caregivers and three acquaintances were aged 65 or older. Among older caregivers, two delivered a partial dose and one delivered a full dose of injectable glucagon. The remaining older caregiver and all three of the older acquaintances were unable to administer any injectable glucagon. In contrast, all seven older study participants administered a full dose of nasal glucagon, suggesting that nasal glucagon is also a better option for use by people aged 65 years or older.

An earlier study of glucagon emergency kits ${ }^{22}$ showed that parents of children and adolescents with diabetes also had difficulty reconstituting and injecting glucagon, even though the parents were familiar with injection techniques and had been trained to administer glucagon. When parents $(N=136)$ were given an unopened emergency glucagon kit and asked to inject glucagon into a simulated thigh, 69\% had difficulties with tasks such as removing the needle sheath and reconstituting the glucagon, as well as problems with unintentionally bending the syringe needles. Six percent aborted the injection entirely. Another 2\% injected only air, and 2\% injected only diluent. Parents who completed the procedure required a mean $2.5 \mathrm{~min}$ to complete the injection, comparable to the time for noninstructed acquaintances in our study. These data indicate that people without medical training may find it challenging to administer injectable glucagon in an emergency situation. The multiple-step process required to reconstitute and inject the glucagon may delay or even prevent treatment.

There are other ongoing developmental efforts to overcome the need for glucagon reconstitution. They include stable liquid glucagon that could be used in a prefilled syringe or an autoinjector. ${ }^{23-26}$ One may anticipate a reduction in the number of errors and time to administration; however, unlike nasal glucagon, these injectable forms of glucagon could still be confused with insulin and may still be problematic for people with a fear of needles.

When comparing glucagon treatments, it is important to consider how quickly they work to raise blood glucose. Rickels et al. showed that intramuscular and nasal glucagon both achieved therapeutic glucagon levels within $5 \mathrm{~min}$ of administration. ${ }^{15}$ At dosing visits with nadir glucose $<50 \mathrm{mg}$ / 
$\mathrm{dL}$, both treatments worked rapidly to increase glucose levels. Indeed, there was only a 3 -min difference $(16 \mathrm{~min}$ vs. 13 min with nasal glucagon and injectable glucagon, respectively $[P<0.001])$ in mean times to reach $70 \mathrm{mg} / \mathrm{dL}$ or to increase by $20 \mathrm{mg} / \mathrm{dL}$. It is worth emphasizing that this study was conducted in a controlled clinical setting and the injectable glucagon was prepared ahead of time, without accounting for the time needed for reconstitution and preparation of the injection.

In a true emergency situation of severe hypoglycemia, the time to glucose elevation is not the only factor in the equation of an effective treatment. For people without medical training, the time needed to reconstitute and inject the glucagon was longer than for nasal glucagon (1.3-3.9 min; Table 4), which may partially or completely offset the 3 -min difference in time of action. Furthermore, the high rate of unsuccessful administration with injected glucagon must be taken into account. In our study, only $13 \%$ of caregivers and none (0\%) of acquaintances administered a full dose of injectable glucagon, compared to $94 \%$ and $93 \%$, respectively, for nasal glucagon. Thus, the significant delay in treatment due to the time needed for emergency medical services to arrive must also be considered.

The majority of people with diabetes (69\%), caregivers $(81 \%)$, and acquaintances $(100 \%)$ would recommend nasal glucagon over injectable glucagon. These data are consistent with those of a telephone survey of patients with T1D, in which most patients indicated that they and their caregivers would prefer nasally administered glucagon to other options. ${ }^{12}$ In our study, three people with diabetes and two caregivers expressed a preference for injectable glucagon because they believed that it would be more effective than nasal glucagon. Study participants were not given any information comparing the efficacy of the two glucagon options; so this preference for injectable glucagon by these caregivers likely came from preconceived thoughts about potential efficacy of nasally delivered versus injectable treatments. In fact, in a controlled clinical research setting, nasal glucagon was shown to be noninferior to injectable glucagon for treatment of hypoglycemia in patients with T1D. ${ }^{15}$

Among the limitations of the study was its nature as a simulation. It may not have fully replicated the fear and emotional distress many people without medical training would experience when treating a person with diabetes experiencing seizure or coma. In addition, the study design resulted in each caregiver or acquaintance encountering the emergency simulation twice; thus, the second situation may have been less stressful. To help control for this, half of these participants used nasal glucagon in the first simulated emergency and half used injectable glucagon, and different stressor elements (i.e., noises, interruptions) were used in the second simulation. In addition, the short memory decay period (1-2 weeks) between when caregivers were instructed, and when they used the treatment option only partially models a real-life delay, which could be months or years. However, results with instructed caregivers and noninstructed acquaintances suggest that instruction only slightly improves the ability to administer injectable glucagon, and is apparently unnecessary for nasal glucagon. Therefore, a longer delay between instruction and an emergency scenario might not have substantially changed the outcome with either delivery system.
In addition, this study assumed that the person suffering from severe hypoglycemia was an adult. Had the simulation focused on a pediatric patient, there would have been the additional complication of having to measure the actual dose, based on age or weight, of injectable glucagon to be administered, according to the product label. ${ }^{27,28}$ Likely, this would have been a source of additional errors. In contrast, a full single dose of nasal glucagon $(3 \mathrm{mg})$ can be administered to children and adolescents, as well as adults. ${ }^{16}$

In conclusion, administration of nasal glucagon is faster and has a much higher success rate for delivery of the full glucagon dose with fewer errors than injectable glucagon. It was preferred over injectable glucagon by both people with diabetes and caregivers. Moreover, administering glucagon using a different route and device form than those used for insulin appears to reduce the risk of accidental delivery of insulin. This suggests that nasal glucagon offers a viable solution to the unmet need for a needle-free glucagon that is easy to use during stressful emergency situations of severe hypoglycemia.

\section{Acknowledgments}

The participation of the people with diabetes, caregivers, and acquaintances in the study is gratefully acknowledged. Assistance with early drafts of this article was provided by David Segarnick, PhD, of MedEvoke (a division of Medisys Health Communications), High Bridge, NJ, and was funded by Locemia Solutions, Montréal, Québec, Canada. Medical writing support for later drafts of this article was provided by Jennie G. Jacobson, PhD, of Eli Lilly and Company, Indianapolis, Indiana, USA. Funding: this study was performed by Core Human Factors, Inc., under contract with Locemia Solutions.

\section{Authors' Contribution}

J.-F.Y., H.D., M.L., D.C., C.A.P., A.R.S., N.K., and M.E. designed the study. R.M., E.D., N.K., H.D., and M.E. conducted the study. All authors were involved in analyzing and interpreting the data as well as drafting and reviewing the article. All authors have approved the final draft of the article for submission.

\section{Author Disclosure Statement}

J.-F.Y. is a consultant to Locemia Solutions, Montréal, Québec, Canada, the developer of Nasal Glucagon. H.D., M.L., D.C., and C.A.P. are employees of Locemia Solutions. R.M., E.D., A.R.S., and M.E. are employees of Core Human Factors, Inc., Bala Cynwyd, Pennsylvania, USA. N.K. is a former employee of Core Human Factors, Inc., Bala Cynwyd, Pennsylvania, USA. M.X.Z. is an employee of Eli Lilly Canada, Inc., Toronto, Ontario, Canada. S.Z. and C.B.G. are employees of Eli Lilly and Company, Indianapolis, Indiana, USA.

\section{References}

1. Kedia N: Treatment of severe diabetic hypoglycemia with glucagon: an underutilized therapeutic approach. Diabetes Metab Syndr Obes 2011;4:337-346.

2. Cryer P: Hypoglycemia in Diabetes: Pathophysiology, Prevalence and Prevention, 2nd ed. Alexandria, VA: American Diabetes Assocation, 2012. 
3. Wild D, von Maltzahn R, Brohan E, et al.: A critical review of the literature on fear of hypoglycemia in diabetes: implications for diabetes management and patient education. Patient Educ Couns 2007;68:10-15.

4. Kovacs Burns K, Nicolucci A, Holt RI, et al.: Diabetes Attitudes, Wishes and Needs second study (DAWN2): cross-national benchmarking indicators for family members living with people with diabetes. Diabet Med 2013;30: 778-788.

5. Wang J, Geiss LS, Williams DE, Gregg EW: Trends in emergency department visit rates for hypoglycemia and hyperglycemic crisis among adults with diabetes, United States, 2006-2011. PLoS One 2015;10:e0134917.

6. Miller KM, Foster NC, Beck RW, et al.: Current state of type 1 diabetes treatment in the US: updated data from the T1D exchange clinic registry. Diabetes Care 2015;38: 971-978.

7. Khunti K, Alsifri S, Aronson R, et al.: Self-reported hypoglycaemia: a global study of 24 countries with 27,585 insulin-treated patients with diabetes: the HAT study. Diabetologia 2014;57(suppl 1):S201:A481.

8. Deacon B, Abramowitz J: Fear of needles and vasovagal reactions among phlebotomy patients. J Anxiety Disord 2006;20:946-960.

9. Nir Y, Paz A, Sabo E, Potasman I: Fear of injections in young adults: prevalence and associations. Am J Trop Med Hyg 2003;68:341-344.

10. Taddio A, Ipp M, Thivakaran S, et al.: Survey of the prevalence of immunization non-compliance due to needle fears in children and adults. Vaccine 2012;30:4807-4812.

11. Polonsky WH, Fisher L, Hessler D, Johnson N: Emotional distress in the partners of type 1 diabetes adults: worries about hypoglycemia and other key concerns. Diabetes Technol Ther 2016;18:292-297.

12. Yanai O, Phillip M, Harman I, et al.: IDDM patients' opinions on the use of glucagon emergency kit in severe episodes of hypoglycaemia. Pract Diabetes 1997;14:40-42.

13. Pontiroli AE: Intranasal glucagon: a promising approach for treatment of severe hypoglycemia. J Diabetes Sci Technol 2015;9:38-43.

14. Boido A, Ceriani V, Pontiroli AE: Glucagon for hypoglycemic episodes in insulin-treated diabetic patients: a systematic review and meta-analysis with a comparison of glucagon with dextrose and of different glucagon formulations. Acta Diabetol 2015;52:405-412.

15. Rickels MR, Ruedy KJ, Foster NC, et al.: Intranasal glucagon for treatment of insulin-induced hypoglycemia in adults with type 1 diabetes: a randomized crossover noninferiority study. Diabetes Care 2016;39:264-270.

16. Sherr JL, Ruedy KJ, Foster NC, et al.: Glucagon nasal powder: a promising alternative to intramuscular glucagon in youth with type 1 diabetes. Diabetes Care 2016;39: $555-562$.

17. Deeb L, Dulude H, Zhang M, et al.: Nasal glucagon (NG) for the treatment of moderate to severe hypoglycemia (hypo) episodes in children and adolescents with type 1 diabetes (T1D) in home or school settings. Pediatric Diabetes 2016;17(Supplement 24):85.

18. Seaquist E, Dulude H, Zhang M, et al.: Nasal glucagon for the treatment of moderate-to-severe hypoglycemic episodes in real-world settings in adults with type 1 diabetes. Diabetes 2017; in press.

19. Davis TC, Crouch MA, Long SW, et al.: Rapid assessment of literacy levels of adult primary care patients. Fam Med 1991;23:433-435.

20. Fries J, Spitz P, Kraines R, Holman H: Measurement of patient outcomes in arthritis. Arthritis Rheum 1980;23: 137-145.

21. Group HPR. NASA Task Load Index (TLX). Moffett Field, CA: NASA Ames Research Center, 1986.

22. Harrism G, Diment A, Sulway M, Wilkinson M: Glucagon administration-underevaluated and undertaught. Practical Diabetes Int 2001;18:22-25.

23. Riber D, Valeur A, Svendgaard M, et al.: The Novel Glucagon Analogue ZP-GA-1 has superior physicochemical properties while maintaining the pharmacokinetic and pharmacodynamic profile of native glucagon. Diabetes 2014;63(Supplement 1):A103.

24. Castle J, El Youssef J, Branigan D, et al.: Comparative pharmacokinetic/pharmacodynamic study of liquid stable glucagon versus lyophilized glucagon in type 1 diabetes subjects. J Diabetes Sci Technol 2016;10:1101-1107.

25. Biochaperone Human glucagon. www.adocia.fr/WP/ products/biochaperone-human-glucagon/ (accessed February 20, 2017).

26. Stable Aqueous Glucagon. www.arecor.com/products/stableaqueous-glucagon/ (accessed February 20, 2017).

27. Information for the user: glucagon for injection (rDNA origin). http://pi.lilly.com/us/rglucagon-ppi.pdf (accessed November 3, 2016).

28. GlucaGen(r) Hypokit(r) instructions for use. www .glucagenhypokit.com/instructions.html (accessed November 3, 2016).

Address correspondence to: Cristina B. Guzman, MD Eli Lilly and Company Lilly Corporate Headquarters Indianapolis, IN 46285

E-mail: guzman_cristina_b@lilly.com 